\title{
A Note on the Intersection of Banach Subspaces
}

\author{
Dedicated to the memory of Klaus Floret
}

By

Gerardo Chacón*, Vicente Montesinos,* and Alfredo OCtavio***

\begin{abstract}
We show that reflexivity of a Banach space can be characterized by a simple property formulated in terms of the distance to the intersection of a decreasing countable family of closed subspaces. We provide some explicit examples of the failure of the property in the non-reflexive case.
\end{abstract}

\section{$\S 1 . \quad$ Introduction}

A Banach space $X$ is reflexive if the canonical injection $j: X \rightarrow X^{* *}$ is an onto mapping. As it is well known, this is equivalent to say that $B_{X}$, the closed unit ball of $X$, is weakly compact. Many other characterizations of reflexivity have been given, maybe the most famous R. C. James' ([J1]) on the normattaining of all elements in $X^{*}$, R. C. James' ([J2]) and independently D. P. Milman and V. D. Milman's ([MM]) on "almost" biorthogonal sequences and Klee's ([Kl], see also [Ko], §24) on separation of disjoint closed convex sets by

Communicated by T. Kawai, June 8, 2000 Revised September 11, 2000.

2000 Mathematics Subject Classification(s): 46B30, 46B03

Key words: Banach spaces, reflexivity.

* Departamento de Ciencias. Universidad de los Andes. Núcleo Táchira.

e-mail: gchacon@cantv.net

Supported in part by CDCHT-ULA (Venezuela).

**Departamento de Matemática Aplicada, E.T.S.I. Telecomunicación, Universidad Politécnica de Valencia. C/Vera, s/n. 46071 Valencia, Spain.

e-mail: vmontesinos@mat.upv.es

Supported in part by Project pb96-0758 (Spain) and Project BFM2002-01423 (Spain).

*** Departamento de Matemáticas. Instituto Venezolano de Investigaciones Científicas. Apartado 21827. Caracas 1020A. Venezuela.

e-mail: aoctavio@ivic.ve 
real hyperplanes (see also, e.g., [F], [LT], [JL] or [FHHMPZ], and the references therein).

In this note we provide another characterization of reflexivity, this time in terms of the distance from a point to a decreasing sequence of closed subspaces. To be precise, let $X$ be a Banach space. Given a non-empty subset $M$ of $X$, denote by $d(x, M):=\inf \{\|x-m\|: m \in M\}$ the distance from $x \in X$ to $M$.

Let $X_{n}, n=1,2,3, \ldots$ be a decreasing sequence of closed subspaces of $X$. We are interested in the validity of the formula

$$
d\left(x, \bigcap_{n=1}^{\infty} X_{n}\right)=\lim _{n \rightarrow \infty} d\left(x, X_{n}\right), \quad x \in X .
$$

We shall prove (Theorem 2.1) that this equation holds if and only if $X$ is a reflexive space. So, if $X$ is not reflexive, there exists a decreasing sequence $\left(X_{n}\right)$ of closed subspaces and a point $x \in X$ such that $\lim _{n \rightarrow \infty} d\left(x, X_{n}\right)<$ $d\left(x, \bigcap_{n=1}^{\infty} X_{n}\right)$. We shall provide in Section 3 , for the sake of completeness, examples of such sequences and points in particular instances.

Our characterization is close to the one given by V. N. Nikolsky [N, p.123] and another one by I. S. Tyuremskih [T, thm. 4] (see also [S], pp.153-154). A Banach space $X$ is said to have property $B_{f}$ if for every increasing sequence

$$
\{0\}=X_{0} \subset X_{1} \subset X_{2} \subset \cdots
$$

of distinct closed linear subspaces of $X$ and every sequence

$$
d_{0} \geq d_{1} \geq d_{2} \geq \cdots \geq d_{n} \geq d_{n+1}=d_{n+2}=\cdots=0,
$$

there exists an element $x \in X$ such that $d\left(x, X_{k}\right)=d_{k}, k=0,1,2, \ldots$

Theorem 1.1 (V. N. Nikolsky $[\mathrm{N}])$. A Banach space is reflexive if and only if it has property $\left(B_{f}\right)$.

Theorem 1.2 (I. S. Tyuremskih [T]). A Banach space $X$ is reflexive if and only if it has the property that for every decreasing sequence of distinct closed linear subspaces

$$
X \supset X_{1} \supset X_{2} \supset \cdots
$$

and every sequence of numbers $\left\{d_{n}\right\}_{n=1}^{\infty}$ such that

$$
0 \leq d_{1} \leq d_{2} \leq \cdots \leq c<\infty
$$

there exists an element $x \in X$ satisfying

$$
\|x\|=\lim _{n \rightarrow \infty} d_{n}, \quad d\left(x, X_{k}\right)=d_{k}, \quad k=1,2, \ldots
$$


We follow the standard notation, as can be found, e.g., in [FHHMPZ].

\section{§2. A Characterization of Reflexivity}

We now state our main result.

Theorem 2.1. $\quad$ A Banach space $X$ is reflexive if and only if the following holds:

Given any $x \in X$ and any decreasing sequence $X_{n}, n=1,2, \ldots$ of closed linear subspaces of $X$,

$$
d\left(x, \bigcap_{n=1}^{\infty} X_{n}\right)=\lim _{n \rightarrow \infty} d\left(x, X_{n}\right) .
$$

Proof. The fact that in the reflexive case equation (1) holds is an easy consequence of the weak lower semicontinuity of the norm and the weak compactness of the closed unit ball.

In order to prove the reverse implication, assume $X$ is separable and nonreflexive. Let $x^{* *} \in X^{* *} \backslash X$ and $x \in S_{X}$ such that $\left\|x^{* *}-x\right\|<1$. Let $H:=$ $\left(x^{* *}\right)^{-1}\{0\} .\left(B_{X^{*}}, w^{*}\right)$ is compact and metrizable, so $\left(B_{H}, w^{*}\right)$ is separable. Let $\left(h_{n}^{*}\right)$ be a dense sequence of $\left(B_{H}, w^{*}\right)$. Define $X_{n}:=\left\{h_{1}^{*}, h_{2}^{*}, \ldots, h_{n}^{*}\right\}_{\perp} \subset X$, $n=1,2, \ldots$ (where $(\cdot)_{\perp}$ denotes the orthogonal set in $\left.X\right)$, so $\left(X_{n}\right)$ is a decreasing sequence of closed subspaces of $X$ and $\bigcap_{n=1}^{\infty} X_{n}=\{0\}$. Now,

$$
d\left(x, X_{n}\right)=\left\|q_{n}(x)\right\|,
$$

where $q_{n}: X \mapsto X / X_{n}$ is the canonical mapping. Observe that $\left(X / X_{n}\right)^{*}$ is isometrically isomorphic to $X_{n}^{\perp}=\operatorname{lin}\left\{h_{1}^{*}, h_{2}^{*}, \ldots, h_{n}^{*}\right\} \subset H$, so

$$
\left\|q_{n}(x)\right\|=\sup \left\{\left|\left\langle x, x^{*}\right\rangle\right| ; x^{*} \in B_{X_{n}^{\perp}}\right\} .
$$

Given $x^{*} \in B_{X_{n}^{\perp}}$

$$
\left|\left\langle x, x^{*}\right\rangle\right|=\left|\left\langle x^{* *}-x, x^{*}\right\rangle\right| \leq\left\|x^{* *}-x\right\|<1,
$$

so $d\left(x, X_{n}\right) \leq\left\|x^{* *}-x\right\|<1, n=1,2, \ldots$ However,

$$
d\left(x, \bigcap_{n=1}^{\infty} X_{n}\right)=d(x,\{0\})=\|x\|=1 .
$$

This proves the result in the separable case. Now, given any nonreflexive Banach space, by the Eberlein-Šmulyan Theorem (see, for example, [FHHMPZ, Thm. 3.59]) there exists a nonreflexive separable subspace and the first part of the proof applies. 


\section{§3. Examples}

We present two non-reflexive Banach spaces and corresponding decreasing sequences of closed subspaces violating equation (1). Both are concrete realizations of the construction in the proof of Theorem 2.1.

Example 1. Let $X$ be the space $c$ of all convergent sequences of real numbers, endowed with the norm $\|x\|:=\|x\|_{\infty}+\lim _{n \rightarrow \infty}\left|x_{n}\right|$. This norm is obviously equivalent to the norm $\|\cdot\|_{\infty}$ induced by $\ell_{\infty}$, so $(X,\|\cdot\|)$ is a Banach space. Let $X_{n}:=\left\{x \in c ; x_{i}=0, i=1,2, \ldots, n\right\}, n=1,2, \ldots\left(\right.$ so $\bigcap_{n=1}^{\infty} X_{n}=$ $\{0\})$. Now $d\left(x, X_{n}\right)=\left\|\left(x_{1}, x_{2}, \ldots, x_{n}, 0,0, \ldots\right)\right\|_{\infty}$, so $\lim _{n \rightarrow \infty} d\left(x, X_{n}\right)=$ $\|x\|_{\infty}$, while $d\left(x, \bigcap_{n=1}^{\infty} X_{n}\right)=\|x\|$. As a consequence,

$$
d\left(x, \bigcap_{n=1}^{\infty} X_{n}\right)>\lim _{n \rightarrow \infty} d\left(x, X_{n}\right)
$$

holds for every $x \notin c_{0}$.

Example 2. The second example concerns spaces of holomorphic functions. Let $\mathcal{W}$ be the space of all Lebesgue integrable complex valued functions $\hat{f}$ which are defined on the unit circle $\mathbb{T}$ and have absolutely convergent series of Fourier coefficients $f(n)$, i.e.

$$
\hat{f}(\zeta)=\sum_{n=-\infty}^{\infty} f(n) \zeta^{n}, \quad \sum_{n=-\infty}^{\infty}|f(n)|<\infty .
$$

The space $\mathcal{W}$ is a subspace of $\mathcal{C}(T)$, the space of continuous functions on $\mathbb{T}$, and it is a Banach space endowed with the norm

$$
\|\hat{f}\|_{\mathcal{W}}=\sum_{n=-\infty}^{\infty}|\hat{f}(n)|
$$

as it is linearly isometric to the Banach space

$$
l_{1}(\mathbb{Z})=\left\{\left\{a_{n}\right\}_{-\infty}^{\infty}: \sum_{n=-\infty}^{\infty}\left|a_{n}\right|<\infty\right\}
$$

(the linear isometry $\left(l_{1}(\mathbb{Z}),\|\cdot\|_{1}\right) \rightarrow\left(\mathcal{W},\|\cdot\|_{\mathcal{W}}\right)$ being the inverse Fourier transform $f \rightarrow \hat{f})$. 
We will use the following notions:

Definition 3.1 ([R, (see, e.g. Def. 5.1.1)]). A subset $E$ of $\mathbb{T}$ is said to be independent if $E$ has the following property: for every choice of distinct points $\zeta_{1}, \ldots, \zeta_{k}$ of $E$ and integers $n_{1}, \ldots, n_{k}$, either

$$
\zeta_{1}^{n_{1}}=\zeta_{2}^{n_{2}}=\cdots=\zeta_{k}^{n_{k}}=1
$$

or

$$
\zeta_{1}^{n_{1}} \zeta_{2}^{n_{2}} \cdots \zeta_{k}^{n_{k}} \neq 1
$$

Definition 3.2 ([R, (see, e.g. Def. 5.6.1)]). A compact subset $P$ in $\mathbb{T}$ is a Helson set if, given $F \in C(P), F=\pi(\hat{f})$ for some $\hat{f} \in \mathcal{W}$, where $\pi: \mathcal{W} \rightarrow C(P)$ is the restriction mapping.

The previous definition is reformulated in the following form (cf. $[\mathrm{R}$, 5.6.2]): For any compact set $P \subset T$ let $\pi: \mathcal{W} \rightarrow C(P)$ be the restriction mapping and $q: \mathcal{W} \rightarrow \mathcal{W} / \operatorname{ker}(\pi)$ the quotient mapping. We have $\|\pi\| \leq 1$. By definition, $P$ is a Helson set if and only if $\pi$ in an onto map. In this case, $\tilde{\pi}: \mathcal{W} / \operatorname{ker}(\pi) \rightarrow C(P)$ is an isomorphism, where $\tilde{\pi} \circ q=\pi$. If $P$ is a countable, compact, independent set in $\mathbb{T}$, then $P$ is a Helson set and it is showed in the proof of $\left[\mathrm{R}\right.$, Th. 5.6.7] that $\left\|\left(\widetilde{\pi}^{*}\right)^{-1}\right\| \leq 2$, where $\widetilde{\pi}^{*}: M(P) \rightarrow(\mathcal{W} / \operatorname{ker}(\pi))^{*}$ is the adjoint mapping of $\tilde{\pi}$ (so $\left\|\widetilde{\pi}^{-1}\right\| \leq 2$ ).

Let $X=\mathcal{W}$ and let $\left\{\zeta_{1}, \zeta_{2}, \ldots\right\}$ be a dense set in $\mathbb{T}$ such that $P_{N}:=$ $\left\{\zeta_{1}, \zeta_{2}, \ldots, \zeta_{n}\right\}$ is an independent set for each $n$. Let

$$
\pi_{n}: \mathcal{W} \rightarrow C\left(P_{n}\right)
$$

be the restriction mapping, as before, and define

$$
X_{n}:=\operatorname{ker}\left(\pi_{n}\right), n=1,2, \ldots
$$

Let $q_{n}: \mathcal{W} \rightarrow \mathcal{W} / X_{n}$ be the canonical mapping.

Since $\left\{\zeta_{j}\right\}_{n=1}^{\infty}$ is dense in $\mathbb{T}$, we have that

$$
\bigcap_{n=1}^{\infty} X_{n}=\{0\} .
$$

The norm $\|\cdot\|_{\mathcal{W}}$ and the supremum norm in $\mathcal{W}$ are not equivalent, so there exist an element $f \in \mathcal{W}$ such that $\|f\|_{\mathcal{W}} \geq 3$, but $\|f\|_{\infty}=\sup _{\zeta \in T}|f(\zeta)|=1$.

Thus

$$
d\left(f, \bigcap_{n=1}^{\infty} X_{n}\right)=\|f\|_{\mathcal{W}} \geq 3 .
$$


On the other side, $\left\|\pi_{n}(f)\right\| \leq 1$, and $d\left(f, X_{n}\right)=\left\|q_{n}(f)\right\|=\|\left(\widetilde{\pi_{n}}\right)^{-1}\left(\pi_{n}(f) \| \leq\right.$ $2\left\|\pi_{n}(f)\right\| \leq 2, n=1,2, \ldots$. Then $f$ and $\left\{X_{n}\right\}$ have the properties that we requested.

Open problem. We do not know of any similar characterization of superreflexive Banach spaces.

\section{Acknowledgements}

We thank the referee for kindly pointing out the reference to Singer's book [S] and Theorems 1.1 and 1.2 appearing there (pages 153-154) and to Prof. López-Pellicer for a remark concerning the proof of Theorem 2.1.

\section{References}

[FHHMPZ] Fabian, M., Habala, P., Hájek, P., Pelant, P., Montesinos, V. and Zizler, V., Infinite Dimensional Topology and Functional Analysis, Canadian Mathematical Society, CMS Books in Mathematics, Springer Verlag, 2001.

[F] Floret, K., Weakly compact sets, $L N M, \mathbf{8 0 1}$, Springer-Verlag, 1980.

[J1] James, R. C., Reflexivity and the supremum of linear functionals, Ann. of Math., 66 (1957), 159-169.

[J2] Characterizations of reflexivity, Studia Math., 23 (1964), 205-216.

[JL] Handbook of the Geometry of Banach Spaces, Vol. I and II, W. B. Johnson and J. Lindenstrauss (Ed.), Elsevier, 2001.

[Kl] Klee, V., Some characterizations of reflexivity, Rev. Ci. Lima, 52 (1950), 15-23.

[Ko] Köthe, G., Topological Vector Spaces I, GMW, 107, Springer-Verlag, 1966.

[LT] Lindenstrauss, J. and Tzafriri, L., Classical Banach Spaces I, EMG, 92, SpringerVerlag 1977.

[MM] Milman, D. P. and Milman, V. D., Some geometric properties of non-reflexive spaces, Soviet Math. Dokl., 4 (1963), 1250-1252.

[N] Nikolsky, V. N., Some properties of reflexive spaces, Ucz. Zap. Kalininsk. gos. pied. in-ta., 29 (1963), 121-125 (in russian).

[R] Rudin, W., Fourier Analysis on Groups, Interscience Publishers, New York, 1967.

[S] Singer, I., Best Approximation in Normed Linear Spaces by elements of Linear Subspaces. Springer-Verlag, New York, 1970.

[T] Tyuremskih, I. S., Some properties of the Tchebychev subspaces of a Banach space, Ucz. Zap. Kalininsk. gos. pied. in-ta., 39 (1964), 53-64 (in russian). 